\title{
Diffusion-weighted MR imaging including bi-exponential fitting for the detection of recurrent or residual tumour after (chemo)radiotherapy for laryngeal and hypopharyngeal cancers
}

\author{
Dechen W. Tshering Vogel • Peter Zbaeren • \\ Andreas Geretschlaeger • Peter Vermathen • \\ Frederik De Keyzer • Harriet C. Thoeny
}

Received: 8 May 2012 /Revised: 25 June 2012 / Accepted: 2 July 2012 /Published online: 4 August 2012

(C) European Society of Radiology 2012

\begin{abstract}
Objectives To assess whether diffusion-weighted magnetic resonance imaging (DW-MRI) including bi-exponential fitting helps to detect residual/recurrent tumours after (chemo)radiotherapy of laryngeal and hypopharyngeal carcinoma.

Methods Forty-six patients with newly-developed/worsening symptoms after (chemo)radiotherapy for laryngeal/ hypopharyngeal cancers were prospectively imaged using conventional MRI and axial DW-MRI. Qualitative (visual assessment) and quantitative analysis (mono-exponentially: total apparent diffusion coefficient $\left[\mathrm{ADC}_{\mathrm{T}}\right]$, and biexponentially: perfusion fraction $\left[\mathrm{F}_{\mathrm{P}}\right]$ and true diffusion coefficient $\left[\mathrm{ADC}_{\mathrm{D}}\right]$ ) were performed. Diffusion parameters
\end{abstract}

D. W. Tshering Vogel $\cdot$ P. Vermathen $\cdot$ H. C. Thoeny $(\bowtie)$

Department of Diagnostic, Interventional and Paediatric

Radiology, Inselspital, University of Bern,

Freiburgstrasse 10,

3010 Bern, Switzerland

e-mail: harriet.thoeny@insel.ch

P. Zbaeren

Department of Oto-Rhino-Laryngology, Head and Neck Surgery,

Inselspital, University of Bern,

Bern, Switzerland

A. Geretschlaeger

Department of Radiation Oncology, Inselspital,

University of Bern,

Bern, Switzerland

F. De Keyzer

Department of Radiology, University Hospitals Leuven,

Leuven, Belgium of tumour versus post-therapeutic changes were compared, with final diagnosis based on histopathology and follow-up. Mann-Whitney $U$ test was used for statistical analysis.

Results Qualitative DW-MRI combined with morphological images allowed the detection of tumour with a sensitivity of $94 \%$ and specificity $100 \% . \mathrm{ADC}_{\mathrm{T}}$ and $\mathrm{ADC}_{\mathrm{D}}$ values were lower in tumour with values $120 \pm 49 \times 10^{-5} \mathrm{~mm}^{2} / \mathrm{s}$ and $113 \pm$ $50 \times 10^{-5} \mathrm{~mm}^{2} / \mathrm{s}$, respectively, compared with posttherapeutic changes with values $182 \pm 41 \times 10^{-5} \mathrm{~mm}^{2} / \mathrm{s}$ $(P<0.0002)$ and $160 \pm 47 \times 10^{-5} \mathrm{~mm}^{2} / \mathrm{s} \quad(P<0.003)$, respectively. $\mathrm{F}_{\mathrm{P}}$ values were significantly lower in tumours than in non-tumours $(13 \pm 9 \%$ versus $31 \pm 16 \%$, $P<0.0002$ ), with $\mathrm{F}_{\mathrm{P}}$ being the best quantitative parameter for differentiation between post-therapeutic changes and recurrence.

Conclusions DW-MRI in combination with conventional MRI substantially improves detection and exclusion of tumour in patients with laryngeal and hypopharyngeal cancers after treatment with (chemo)radiotherapy on both qualitative and quantitative analysis, with $F_{P}$ being the best quantitative parameter in this context.

Key Points

- DW-MRI is increasingly used to detect tumour recurrence.

- DW-MRI allows accurate post-treatment recurrence detection in laryngeal or hypopharyngeal cancer

- $A D C$ values in recurrent tumour are lower than in benign tissue alterations

- Both qualitative and quantitative DW-MRI approaches allow detection of recurrence

- DW-MRI can easily be added to daily clinical routine imaging 
Keywords (Chemo)radiotherapy · Cancer · Diffusionweighted MRI · Apparent diffusion coefficient · Perfusion fraction

\section{Introduction}

Patients with cancer of the larynx and hypopharynx are increasingly treated with (chemo)radiotherapy, aiming to preserve organ function and improve quality of life $[1,2]$. However, it is well known that (chemo)radiotherapy, in addition to its anti-tumoral effects, also results in local oedema, inflammation, fibrosis and, less commonly, necrosis [2-4]. These secondary changes pose a problem, not only for the patient's quality of life, but also for the posttreatment assessment of anti-tumour efficacy. Although detection of residual/recurrent tumour against this background is very challenging and a well-known diagnostic dilemma for both clinicians and radiologists [1, 4-8], early tumour assessment can increase the chance of curative salvage surgery, sometimes allowing preservation of laryngeal function $[4,5]$. Clinical evaluation of possible post-treatment recurrence is difficult owing to oedema obstructing evaluation of the larynx, and owing to the swelling and induration of the soft tissues preventing adequate palpation. Moreover, imaging evaluation is also hampered, as enhancing benign focal mass-like lesions can mimic residual/recurrent tumour [3], leading to possible false-positive diagnosis and unnecessary surgery [9]. Adversely, some residual or recurrent tumours do not enhance after contrast medium administration, further confounding the issue [10].

The most reliable method of detecting recurrent tumour was considered to be direct laryngoscopy under general anaesthesia [5], but this technique is limited as it can miss recurrent tumour directly under the mucosa or wrongly assess the multifocal nature of the tumour. While a positive biopsy is conclusive, a negative biopsy is not sufficient to exclude tumour $[2,6]$. A non-invasive imaging test allowing accurate and comprehensive post-treatment lesion characterisation could therefore be used to select those patients who might benefit from a laryngoscopy, thereby limiting unnecessary biopsies and patient morbidity, and may even indicate the most appropriate biopsy location.

Diffusion-weighted magnetic resonance imaging (DW-MRI) has only recently been introduced in the evaluation of head and neck cancers [11]. It is based on the relative mobility of water molecules in different tissues and provides information about the microenvironment such as cell density, cell integrity and vascularity. Initial results suggest that this non-invasive test may be more specific than FDG-PET in the detection of tumour recurrence or persistence after treatment of various head and neck tumours [7, 12].
The present prospective study is aimed at assessing the ability of qualitative and quantitative DW-MRI, including bi-exponential fitting based on the intravoxel incoherent motion theory $[13,14]$, to detect residual/recurrent tumour against the background of treatment-induced changes in a well-defined group of patients with laryngeal and hypopharyngeal tumours after (chemo)radiotherapy.

\section{Methods and materials}

\section{Approval}

The local ethics committee approved the study protocol and written informed consent was obtained from all participants.

\section{Patients}

Fifty consecutive patients with laryngeal or hypopharyngeal cancers, presenting with newly-developed or worsening symptoms after (chemo)radiotherapy, between May 2007 and December 2010, prospectively underwent MRI of the neck to exclude residual/recurrent tumour. (Chemo)radiotherapy was performed with total doses of 66-72 Gy (5×2 Gy/week). Patients' symptoms included pain, stridor, increasing hoarseness, and breathing and swallowing difficulties. Four patients were excluded owing to susceptibility artefacts compromising image quality, leaving a group of 46 patients (41 men, 5 women, median age 61 years, range 41-83 years) with laryngeal $(n=30)$ or hypopharyngeal cancer $(n=16)$. Of the 30 patients with laryngeal cancers, 11 were staged as T1, 10 as T2 and 9 as T3. Of the 16 patients with hypopharyngeal cancer, 4 were staged as T1 disease, 6 as T2, 2 as T3 and 4 as T4. All patients were treated with fractionated radiotherapy: alone $(n=17)$, or after surgery of the larynx $(n=4)$, or alongside chemotherapy with either methotrexate or cisplatin $(n=25)$. Nine patients also had a neck dissection. All patient and tumour characteristics are presented in Table 1.

\section{MRI}

MRI was performed on a 1.5-T unit (Sonata; Siemens, Erlangen, Germany) using a dedicated neck coil. The conventional MRI included an axial T1-weighted turbospinecho (TSE) sequence (slice thickness, $3 \mathrm{~mm} ; 24$ slices; intersection gap, $0.6 \mathrm{~mm}$; repetition time/echo time [TR/ TE], $624 \mathrm{~ms} / 12 \mathrm{~ms}$; matrix, $512 \times 256$; field of view [FoV], $280 \times 170 \mathrm{~mm}$ ) and an axial T2-weighted TSE sequence (slice thickness, $3 \mathrm{~mm}$; 24 slices; intersection gap, $0.6 \mathrm{~mm}$; TR/TE, $3,630 \mathrm{~ms} / 76 \mathrm{~ms}$; matrix, $512 \times 256$; FoV, $280 \times 170 \mathrm{~mm}$ ), covering the larynx. After gadolinium 
Table 1 Patient and tumour characteristics

\begin{tabular}{|c|c|c|c|c|c|c|c|c|c|}
\hline \multicolumn{2}{|c|}{$\begin{array}{l}\text { Patient, } \\
\text { age and sex }\end{array}$} & \multicolumn{2}{|l|}{$\begin{array}{l}\text { Tumour and TNM } \\
\text { classification }\end{array}$} & \multirow{2}{*}{$\begin{array}{l}\text { Therapy } \\
\text { RT, PL }\end{array}$} & \multirow{2}{*}{$\begin{array}{l}\text { RT dose } \\
\text { (Gy) } \\
66\end{array}$} & \multirow{2}{*}{$\begin{array}{l}\text { Time between MRI and } \\
\text { histology (months) }\end{array}$} & \multirow{2}{*}{$\begin{array}{l}\text { Time between RT } \\
\text { and MRI (months) }\end{array}$} & \multirow{2}{*}{$\begin{array}{l}\text { Tumour based } \\
\text { on Histo }\end{array}$} & \multirow[t]{2}{*}{$\begin{array}{l}\text { FU duration and } \\
\text { type (months) }\end{array}$} \\
\hline P1 & $54 \mathrm{M}$ & Transglottic & T2N0 & & & & & & \\
\hline $\mathrm{P} 2$ & $62 \mathrm{M}$ & Piriform sinus & T4N2 & CRT & 72 & 1 & 3 & + & \\
\hline P3 & $54 \mathrm{M}$ & Glottic & T1N0 & RT & 68 & $<1$ & 60 & + & \\
\hline $\mathrm{P} 4$ & $76 \mathrm{M}$ & Glottic & T1N0 & RT & 68 & $<1$ & 108 & + & \\
\hline P5 & $61 \mathrm{M}$ & Piriform sinus & $\mathrm{T} 1 \mathrm{~N} 2$ & CRT, ND & 64 & $<1$ & 84 & + & \\
\hline P6 & $52 \mathrm{M}$ & Supraglottic & T3N0 & CRT & 72 & $<1$ & 36 & + & \\
\hline P7 & $72 \mathrm{M}$ & Supraglottic & T2N0 & CRT & 72 & 2 & $55,64,69,72$ & + & \\
\hline P8 & $81 \mathrm{M}$ & Glottic & T2N0 & CRT & 70 & 2 & 24 & + & \\
\hline P9 & $67 \mathrm{M}$ & Glottic & T1N0 & RT & 68 & 2 & 12 & + & \\
\hline $\mathrm{P} 10$ & $58 \mathrm{M}$ & Glottic & T1N0 & RT & 68 & 2 & 6,8 & + & \\
\hline $\mathrm{P} 11$ & $51 \mathrm{M}$ & Glottic & T1N0 & RT & 68 & 1 & 46,48 & + & \\
\hline $\mathrm{P} 12$ & $62 \mathrm{M}$ & Piriform sinus & T1N0 & CRT & 72 & 1 & 6 & + & \\
\hline $\mathrm{P} 13$ & $54 \mathrm{~F}$ & Supraglottic & $\mathrm{T} 3 \mathrm{~N} 2$ & CRT, ND & 72 & $<1$ & 7 & + & \\
\hline $\mathrm{P} 14$ & $66 \mathrm{M}$ & Glottic & T2N0 & RT & 72 & $<1$ & 48 & + & \\
\hline $\mathrm{P} 15$ & $83 \mathrm{M}$ & Glottic & T1N0 & RT & 72 & 1 & 60 & + & \\
\hline P16 & $61 \mathrm{M}$ & Supraglottic & T3N3 & RT, ND & 72 & 1 & 27 & + & \\
\hline $\mathrm{P} 17$ & $65 \mathrm{M}$ & Supraglottic & T3N0 & CRT & 72 & $<2$ & 7 & + & \\
\hline $\mathrm{P} 18$ & $66 \mathrm{M}$ & Glottic & T2N0 & CRT & 72 & 1 & 64 & + & \\
\hline P19 & $51 \mathrm{~F}$ & Supraglottic & T2N0 & RT, LR & 72 & $<1$ & 19,24 & - & $48 \mathrm{CT}$ \\
\hline $\mathrm{P} 20$ & $60 \mathrm{~F}$ & Supraglottic & T2N0 & CRT & 72 & 5 & 8 & - & $18 \mathrm{Cl}$ \\
\hline $\mathrm{P} 21$ & $53 \mathrm{M}$ & Glottic & T1N0 & RT & 68 & 2 & 14 & - & $36 \mathrm{MRI}$ \\
\hline $\mathrm{P} 22$ & $55 \mathrm{M}$ & Piriform sinus & T2N0 & CRT & 72 & 1 & $12,47,56$ & - & $32 \mathrm{MRI}$ \\
\hline $\mathrm{P} 23$ & $61 \mathrm{M}$ & Supraglottic & $\mathrm{T} 2 \mathrm{~N} 1$ & CRT, ND & 72 & 2 & 8 & - & $13 \mathrm{Cl}$ \\
\hline $\mathrm{P} 24$ & $61 \mathrm{M}$ & Supraglottic & T3N0 & RT & 72 & 1 & $7,10,28$ & - & $23 \mathrm{MRI}$ \\
\hline $\mathrm{P} 25$ & $41 \mathrm{M}$ & Piriform sinus & $\mathrm{T} 2 \mathrm{~N} 0$ & CRT & 72 & 1 & $69,80,96$ & - & $29 \mathrm{CT}$ \\
\hline P26 & $65 \mathrm{M}$ & Transglottic & T3N0 & CRT & 72 & 10 & 14 & - & $18 \mathrm{Cl}$ \\
\hline $\mathrm{P} 27$ & $45 \mathrm{M}$ & Piriform sinus & T4N0 & CRT & 72 & & $6,9,15,28,33$ & & $39 \mathrm{MRI}$ \\
\hline P28 & $60 \mathrm{M}$ & Transglottic & T3N0 & CRT & 72 & & 50 & & $17 \mathrm{Cl}$ \\
\hline P29 & $73 \mathrm{M}$ & Glottic & T1N0 & RT & 68 & & 75 & & $12 \mathrm{MRI}$ \\
\hline P30 & $69 \mathrm{M}$ & Piriform sinus & $\mathrm{T} 2 \mathrm{~N} 1$ & CRT & 72 & & 2,9 & & $15 \mathrm{CT}$ \\
\hline $\mathrm{P} 31$ & $52 \mathrm{M}$ & Piriform sinus & T3N2 & CRT & 72 & & 26 & & $12 \mathrm{Cl}$ \\
\hline P32 & $72 \mathrm{M}$ & Piriform sinus & $\mathrm{T} 2 \mathrm{~N} 1$ & CRT, ND & 72 & & 8,14 & & $12 \mathrm{Cl}$ \\
\hline P33 & $66 \mathrm{M}$ & Piriform sinus & $\mathrm{T} 2 \mathrm{~N} 1$ & RT & 72 & & 22 & & $24 \mathrm{Cl}$ \\
\hline P34 & $63 \mathrm{M}$ & Piriform sinus & T1N0 & RT, LR, ND & 72 & & $6,9,11,15$ & & $24 \mathrm{Cl}$ \\
\hline P35 & $65 \mathrm{M}$ & Supraglottic & T1N0 & CRT & 72 & & 14,31 & & $12 \mathrm{Cl}$ \\
\hline P36 & $53 \mathrm{M}$ & Hypopharynx wall & T1N0 & RT & 72 & & $44,50,58$ & & $12 \mathrm{Cl}$ \\
\hline P37 & $53 \mathrm{~F}$ & Supraglottic & T2N0 & CRT, ND & 72 & & 9 & & $14 \mathrm{MRI}$ \\
\hline P38 & $57 \mathrm{M}$ & Hypopharynx wall & T4N2 & RT & 72 & & 108 & & $18 \mathrm{Cl}$ \\
\hline P39 & $55 \mathrm{~F}$ & Supraglottic & T1N0 & RT & 72 & & $3,9,16$ & & $12 \mathrm{Cl}$ \\
\hline P40 & $65 \mathrm{M}$ & Piriform sinus & $\mathrm{T} 3 \mathrm{~N} 2$ & RT, LR, ND & 72 & & 11,20 & & 17 MRI \\
\hline P41 & $53 \mathrm{M}$ & Piriform sinus & T4N2 & CRT & 72 & & 3 & & $18 \mathrm{MRI}$ \\
\hline P42 & $66 \mathrm{M}$ & Supraglottic & $\mathrm{T} 2 \mathrm{~N} 1$ & CRT & 72 & & 5 & & $23 \mathrm{CT}$ \\
\hline P43 & $41 \mathrm{M}$ & Supraglottic & T3N2 & RT & 72 & & 3,10 & & $14 \mathrm{Cl}$ \\
\hline P44 & $53 \mathrm{M}$ & Glottic & T1N0 & CRT & 72 & & 35,48 & & $24 \mathrm{CT}$ \\
\hline P45 & $46 \mathrm{M}$ & Supraglottic & T3N2 & CRT, ND & 72 & & 6,10 & & $36 \mathrm{CT}$ \\
\hline P46 & $63 \mathrm{M}$ & Piriform sinus & $\mathrm{T} 2 \mathrm{~N} 1$ & $\mathrm{RT}$ & 72 & & 20 & & $24 \mathrm{Cl}$ \\
\hline
\end{tabular}

$M R I$ magnetic resonance imaging, $R T$ radiotherapy, Histo histopathology, $F U$ follow-up, $M$ male, $P L$ partial laryngectomy, + positive, $C R T$ (chemo)radiotherapy, $F$ female, $N D$ neck dissection, $L R$ laser resection, - negative, $C T$ computed tomography, $C l$ clinical 
injection, T1-weighted fat-saturated sequences were performed in the axial plane (using identical parameters as precontrast medium administration) and in the coronal or sagittal plane (19 slices; slice thickness, $3 \mathrm{~mm}$; intersection gap, $0.75 \mathrm{~mm}$; TR/TE, $630 \mathrm{~ms} / 18 \mathrm{~ms}$; matrix, $512 \times 256$; field of view, $320 \times 250 \mathrm{~mm}$ ). Echo-planar DW-MRI was performed in the axial plane, using the above-mentioned slice geometry, and 4 signals acquired, TR/TE, 3,500 ms/69 ms; matrix, $128 \times 104$; FoV, $290 \times 290 \mathrm{~mm}$; bandwidth $2,056 \mathrm{~Hz}$ per pixel. Six diffusion gradient $b$ values $(0,50,100,500,750$ and $1,000 \mathrm{~s} / \mathrm{mm}^{2}$ ) were applied in three orthogonal directions, minimising the effects of diffusion anisotropy.

Image analysis

Conventional MR images were evaluated on an EasyVision picture archiving and communication system workstation (Philips Medical Systems, Netherlands). Baseline computed tomography (CT) and MRI, as well as repeat examinations after treatment, were available for all patients. CT and MRI examinations were evaluated by two experienced radiologists in consensus (first and last authors, 7 and 12 years of experience in head and neck radiology, respectively). Changes at the tumour site, tissue swelling (focal or diffuse), size changes and enhancement pattern were assessed. Focal areas of enhancement increasing in size were suspicious for tumour, whereas size decrease indicating treatment response and absence of growth on follow-up imaging was considered compatible with post-therapeutic changes, although small residual tumour foci could not be excluded.

The qualitative DW-MRI analysis was performed in conjunction with the conventional images as the poor spatial resolution of DW-MRI precludes interpretation on its own. DW-MR images at a b value of $1,000 \mathrm{~s} / \mathrm{mm}^{2}$ (b-1,000 images) and the corresponding apparent diffusion coefficient (ADC) maps were matched to and evaluated with the morphological images. The primary tumour site was evaluated. Hyperintense signal on the b-1,000 image compared with the surrounding tissue with corresponding low signal intensity in the matching ADC map was considered positive for tumour (Fig. 1). High signal intensity on b-1,000 images with corresponding high signal on the matching ADC map was considered to represent T2 shine-through and therefore no tumour (Fig. 2). Absence of hyperintensity on the b1,000 image was also considered negative for tumour (Fig. 3).

Quantitative DW-MRI analysis was performed on a pixelby-pixel basis in two ways as previously described [13, 14], yielding total $\mathrm{ADC}\left(\mathrm{ADC}_{\mathrm{T}}\right)$ by mono-exponential fitting, and the perfusion fraction $\left(\mathrm{F}_{\mathrm{P}}\right)$ and true diffusion coefficient $\left(\mathrm{ADC}_{\mathrm{D}}\right)$ by bi-exponential fitting. Using mono-exponential fitting, the $\mathrm{ADC}_{\mathrm{T}}$ value is calculated from all the data obtained, ignoring different contributions of diffusion and perfusion, which is most commonly used in the literature. Acquiring multiple $b$ values allows separating diffusion and micro-perfusion contributions by bi-exponential fitting. This yields $\mathrm{F}_{\mathrm{P}}$, which represents the contribution of microcirculation of blood and movement in pre-defined structures to the signal decay and the diffusion component $\mathrm{ADC}_{\mathrm{D}}$, which mainly reflects true diffusion [14].

Elliptical regions of interest (ROIs) were placed on areas of diffusion impediment taking care to exclude areas of obvious necrosis. If no areas showed diffusion restriction on the b-1000 images, ROIs were placed at or near the
Fig. 1 MR images of an 81year-old man with hoarseness after radiotherapy for a laryngeal tumour. Axial T1-weighted (a) and T2-weighted images (b) showing heterogeneous signal at the right thyroid cartilage and surrounding soft tissues. A focal bright area is visible on the axial b-1,000 image (c) with hypointense signal on the corresponding ADC map (d) indicating focal diffusion impediment, implying tumour (arrows)
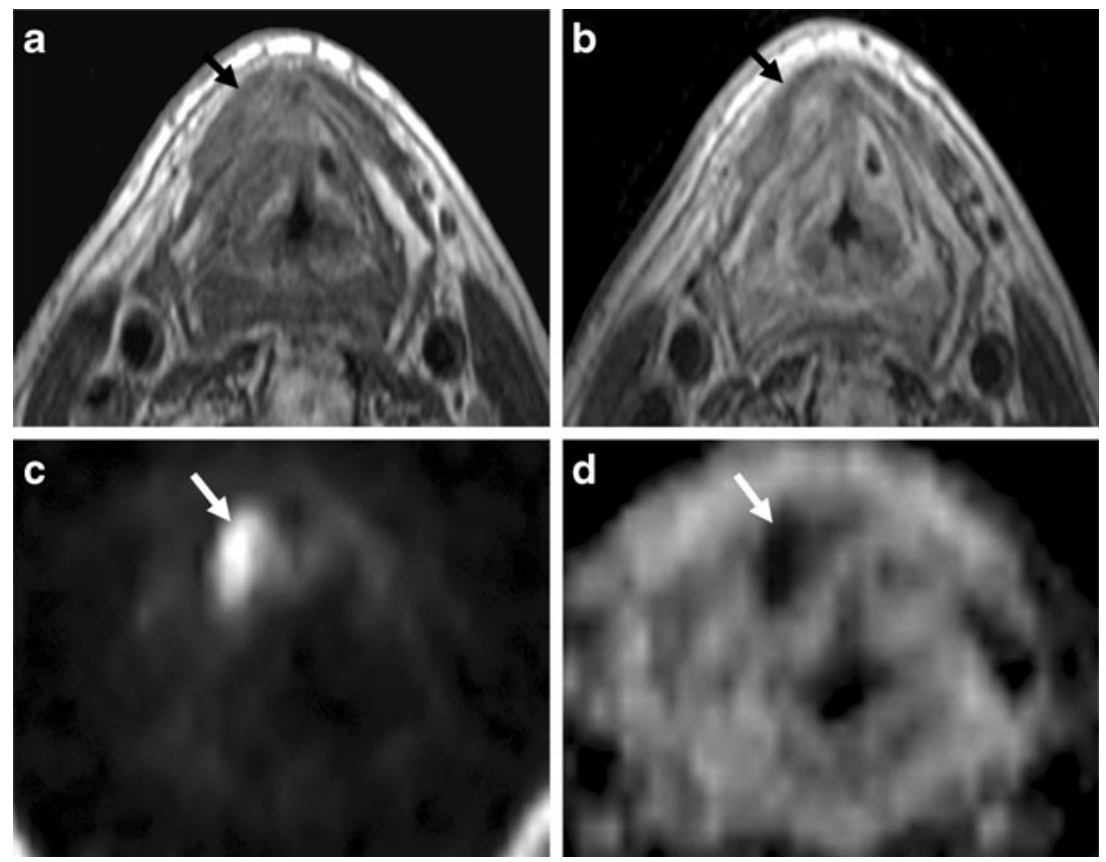
Fig. 2 MR images of a 61year-old man with hoarseness after radiotherapy for a laryngeal tumour. Axial T1-weighted (a) and T2-weighted images (b) showing diffuse and asymmetric swelling of the aryepiglottic folds and hypopharynx in this patient treated for cancer of the right piriform sinus. The b1,000 image (c) shows hyperintense signal, with hyperintensity in the corresponding ADC map (d) representing T2 shinethrough, correlating with posttherapeutic changes (arrows)
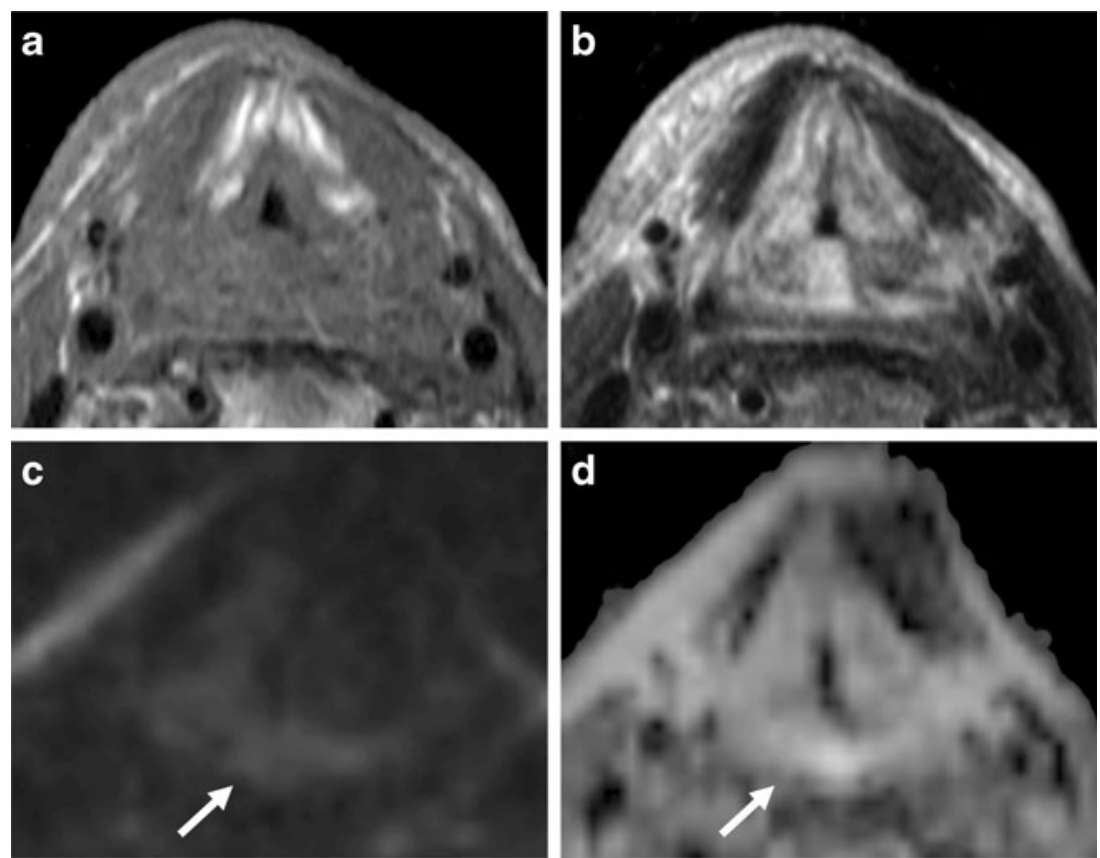

original site of the tumour on the basis of the morphological images regardless of the signal intensity on the DW-MR images. The ROI size varied according to the size of the suspicious lesion (mean size $124 \pm 53 \mathrm{~mm}^{3}$ ).

\section{Histopathology}

Imaging results were compared with histopathology and follow-up. Eighteen patients had histopathologically proven tumour (six laryngectomy specimens and 12 biopsies). Twenty-eight patients did not have a tumour with at least
1 year follow-up after the MR study, and negative histology (biopsy) was also available in 8 of these patients (Table 1).

\section{Statistical analysis}

Statistical analysis was performed with a Mann-Whitney $U$ test to compare patients with recurrent tumour with those with benign alterations. A $P$ value of less than 0.05 was considered statistically significant. Box-whisker plots were used to look at group-wise differences in quantitative diffusion parameters, and optimal cut-off values were determined
Fig. 3 MR images of a 65-yearold man with hoarseness after radiotherapy for a laryngeal tumour. Axial fat-saturated T1weighted images (a) after contrast medium injection showing cricoid cartilage destruction on the right side (arrow) in this patient treated for a transglottic tumour. The patient also had a tracheostoma (star). The 6month follow-up axial fatsaturated T1-weighted image after contrast medium injection (b) shows increasing destruction of the cricoid cartilage on the right (arrow). The b-1,000 image (c) and ADC map (d) at that time did not show diffusion restriction. Biopsy and follow-up at 18 months were negative
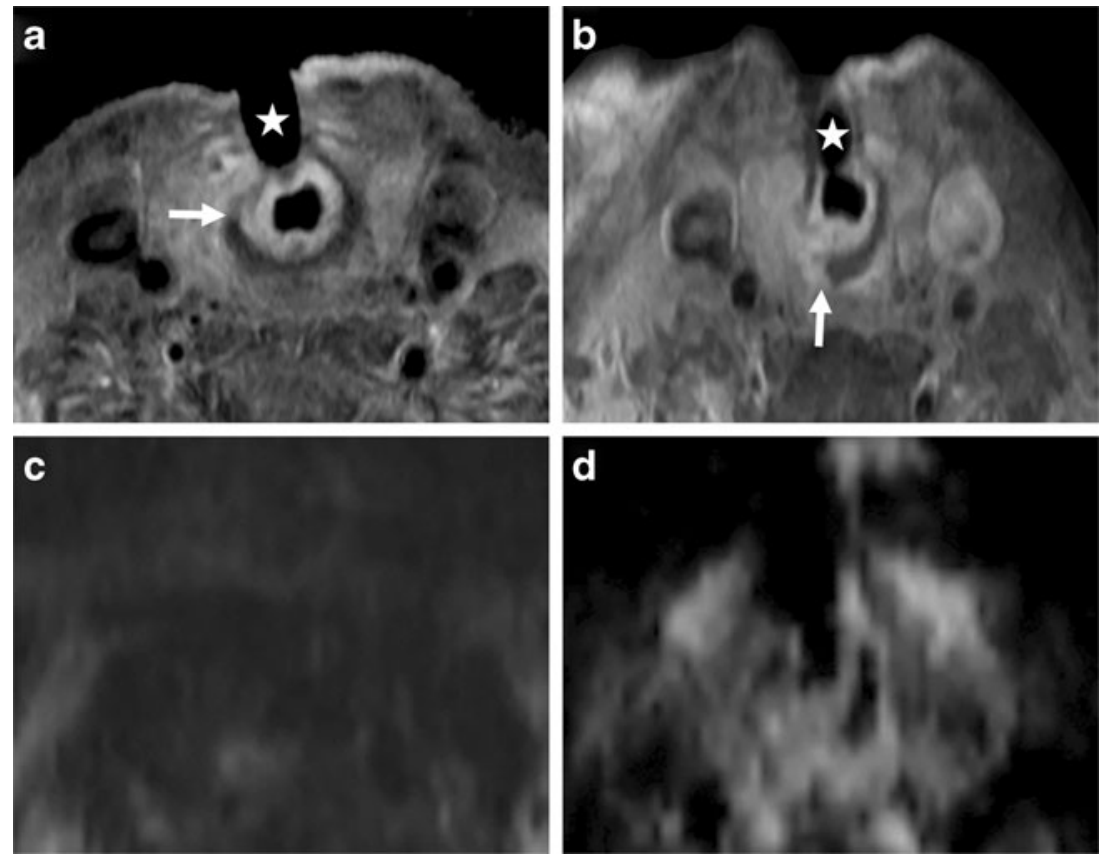
using receiver operating characteristics (ROC) curves, attributing equal weight to sensitivity and specificity. Statistical analysis was performed with SPSS, version 12.0.1 (SPSS, Chicago, IL, USA) and Excel 2002 (Microsoft, Redmond, WA, USA).

\section{Results}

Conventional MR images showed various degrees of posttherapeutic anatomical distortion with most patients showing an asymmetrical swelling. A focal swelling and enhancement was seen in 13 out of 18 patients with histologically proven tumour and in 12 out of 28 patients without tumour (sensitivity $72 \%$, specificity $57 \%$ and accuracy $63 \%)$.

By adding the qualitative $D W-M R I$ analysis, it was possible to exclude tumour in all patients without tumour and to detect recurrence/residual tumour in 17 out of 18 patients with tumour. DW-MRI did not show diffusion restriction in one patient with recurrent tumour, resulting in one falsenegative study (sensitivity 94\%, specificity $100 \%$ and accuracy $98 \%$ ).

All patients diagnosed with tumour underwent a biopsy within 2 months of the MRI. Only six patients without a suspected tumour underwent a biopsy within 2 months of the DW-MRI study, one patient after
5 months and one after 10 months, based on clinical grounds. The biopsies in patients without suspicion of tumour were performed early in the study when the confidence level in DW-MRI was low. With increasing experience and higher confidence in DW-MRI, unnecessary biopsies were avoided in 20 patients without tumour. All these patients were closely followed and did not present with recurrent tumour for at least 1 year after the MR study.

The results of the quantitative analysis with the $\mathrm{ADC}_{\mathrm{T}}, \mathrm{ADC}_{\mathrm{D}}$ and $\mathrm{F}_{\mathrm{P}}$ from mono- and bi-exponential fitting are presented in Fig. 4. $\mathrm{ADC}_{\mathrm{T}}$ values were lower for patients with tumour (mean $120 \pm 49 \times 10^{-5} \mathrm{~mm}^{2} / \mathrm{s}$ ) compared with those without tumour (mean $182 \pm 41 \times$ $\left.10^{-5} \mathrm{~mm}^{2} / \mathrm{s} ; P<0.0002\right)$. ROC analysis provided an optimal threshold for $A D C_{T}$ of $130 \times 10^{-5} \mathrm{~mm}^{2} / \mathrm{s}$ resulting in a sensitivity of $67 \%$, specificity of $86 \%$ and accuracy of $78 \%$. Similarly, $\mathrm{ADC}_{\mathrm{D}}$ values were significantly lower for patients with tumour (mean $113 \pm 50 \times$ $10^{-5} \mathrm{~mm}^{2} / \mathrm{s}$ ) than for those without tumour (mean $160 \pm$ $\left.47 \times 10^{-5} \mathrm{~mm}^{2} / \mathrm{s} ; P<0.003\right)$. For the $A D C_{D}$, a threshold value of $130 \times 10^{-5} \mathrm{~mm}^{2} / \mathrm{s}$ results in a sensitivity of $78 \%$, specificity of $71 \%$ and accuracy of $74 \% . \mathrm{F}_{\mathrm{P}}$ values were significantly reduced in tumours compared with non-tumours $(13 \pm 9 \%$ versus $31 \pm 16 \%, P<0.0002)$. For $\mathrm{F}_{\mathrm{P}}$ a threshold value of $23 \%$ results in a sensitivity of $94 \%$, specificity of $71 \%$ and accuracy of $80 \%$. a
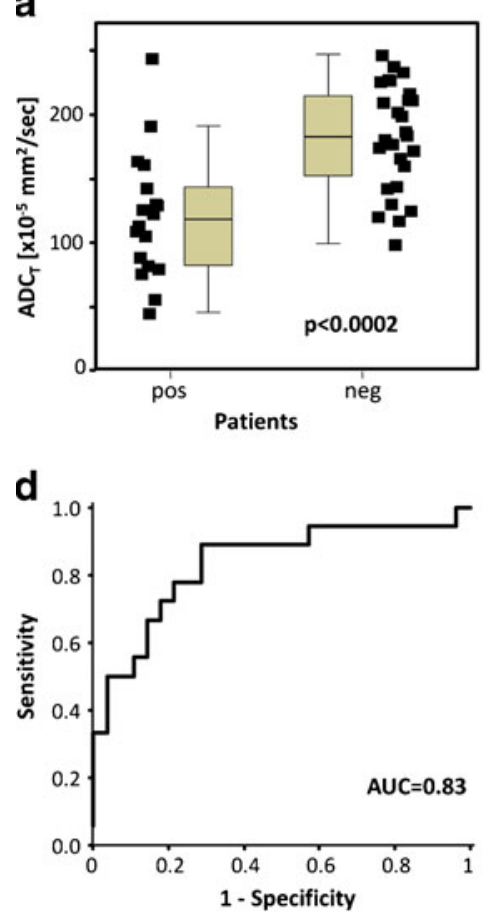

b
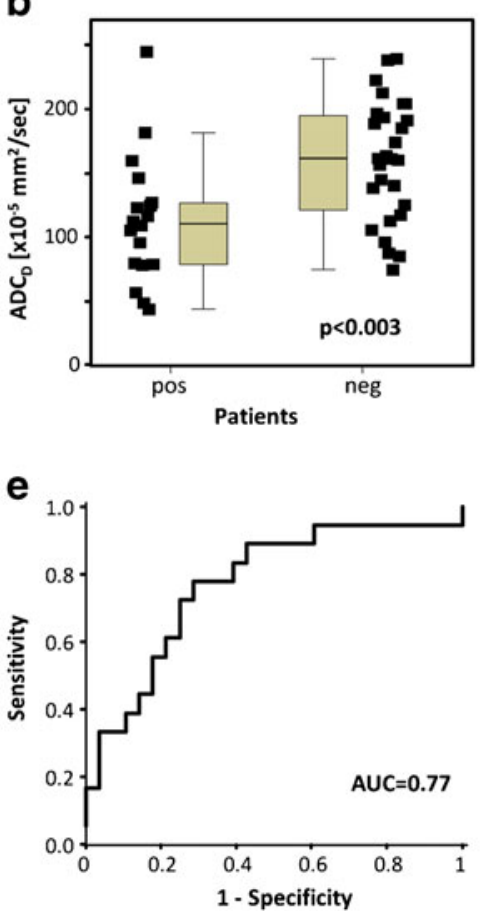

C

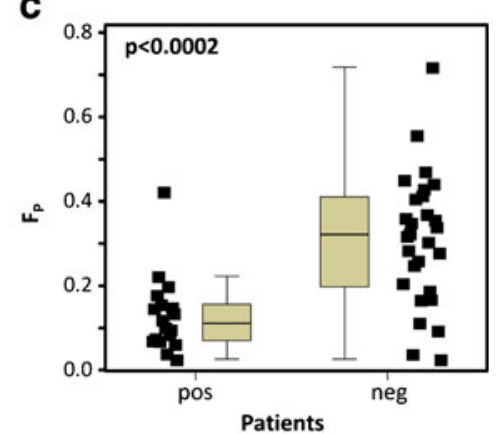

f

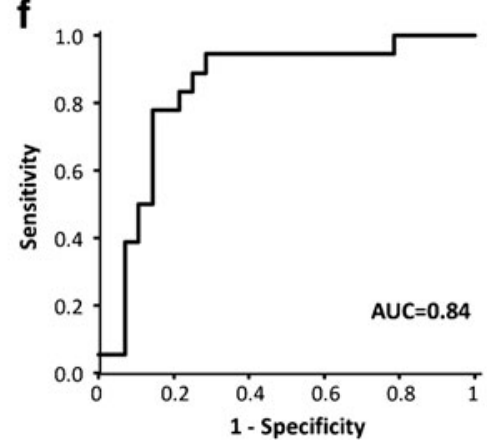

Fig. 4 Box-whisker charts showing the distribution of values of the quantitative parameters $\mathrm{ADC}_{\mathrm{T}}(\mathbf{a}), \mathrm{ADC}_{\mathrm{D}}(\mathbf{b})$ and $\mathrm{F}_{\mathrm{P}}(\mathbf{c})$ in patients with (pos) and without (neg) tumour. Corresponding ROC curves for the qualitative parameters showing the area under the curve $(A U C)$ for $\mathrm{ADC}_{\mathrm{T}}(\mathbf{d}), \mathrm{ADC}_{\mathrm{D}}(\mathbf{e})$ and $\mathrm{F}_{\mathrm{P}}(\mathbf{f})$ 


\section{Discussion}

The present prospective study in a well-defined group of patients with laryngeal or hypopharyngeal cancer treated with (chemo)radiotherapy shows that MRI including DWMRI allows detection or exclusion of tumour with high accuracy. Based on qualitative analysis using visual assessment of high b-value images and the respective ADC maps, tumour could be correctly excluded in all patients without recurrence, while a recurrent tumour was missed in only one patient. These findings are important in daily clinical routine as a DW-MRI sequence can easily be added to any MR protocol without an undue time penalty. Thus, DW-MRI overcomes the diagnostic dilemma of recurrence versus post-therapeutic changes allowing correct tumour detection against a background of treatment-induced soft tissue alteration. Correlation with morphological images in order to accurately localise the findings and reader experience in DW-MRI are additional prerequisites for successful interpretation of this functional imaging technique. However, in some cases image quality of DW-MRI can be reduced due to susceptibility artefacts impeding image interpretation.

Several studies have already shown the benefit of DWMRI in detecting residual or recurrent tumour in head and neck squamous cell cancer, but included tumours of various anatomical regions and focused on quantitative image interpretation. Our results using visual analysis and the $\mathrm{ADC}_{\mathrm{T}}$ are in agreement with a short communication [7] on DWMRI in four patients with laryngeal cancer after radiotherapy, where lower ADC in tumour recurrence than in benign post-therapeutic alterations has been reported. The quantitative values in our study were also very similar to a study on 32 patients with various head and neck tumours [10] that found ADC values for residual or recurrent tumour of $117 \times$ $10^{-5} \mathrm{~mm}^{2} / \mathrm{s}$ and for post-treatment changes of $207 \times$ $10^{-5} \mathrm{~mm}^{2} / \mathrm{s}$, even though we only included patients with laryngeal and hypopharyngeal cancers. The authors of that study found an identical optimal ADC cut-off, and reported a broad range of overlap, comparable to the current study. However, in that study the overlap of ADC values might also be due to sampling error of the reference standard biopsy, whereas in our study we provided a follow-up of at least 1 year in all negative biopsies.

Another study reported a sensitivity of $95 \%$, specificity of $96 \%$ and accuracy of $95.5 \%$, again using the same ADC threshold of $130 \times 10^{-5} \mathrm{~mm}^{2} / \mathrm{s}$, in differentiating between residual or recurrent tumour and post-therapeutic changes [12] in 26 patients with various head and neck cancers. The $\mathrm{b}$ values used and the resulting ADC values (111 and $185 \times$ $10^{-5} \mathrm{~mm}^{2} / \mathrm{s}$ for tumour and benign alterations, respectively) were nearly identical to ours. Thus, we corroborate these findings in a larger patient population but including only a well-defined group of patients with laryngeal and hypopharyngeal tumours. We did find a larger overlap between benign and malignant outcomes, most likely due to the more difficult imaging site of the larynx (susceptibility and movement artefacts), compared with other locations in the head and neck, the overall smaller tumour size in our study ( 15 of the 46 patients were classified as T1, compared with 7 out of 26 in the above-mentioned study), and their inclusion of lymph nodes in the analysis.

The overlap in diffusion values could be caused by partial volume effects due to tiny structures, falsely lowered ADC in fibrosis and falsely increased ADC in diffuse necrotic parts of recurrent tumour. Furthermore, the $\mathrm{ADC}$ and $\mathrm{F}_{\mathrm{P}}$ values depend on the ROI placement during visual analysis, and are therefore linked to observer experience. The complex structure of the larynx with many different tissues, including mucosa, cartilage (ossified or non-ossified), muscle, fat and air in close proximity can also lead to susceptibility effects and impaired image contrast, further hampering correct $\mathrm{ADC}$ measurements.

In order to improve quantitative image interpretation, we also provided information on bi-exponential fitting $\left(\mathrm{ADC}_{\mathrm{D}}\right.$ and $\mathrm{F}_{\mathrm{P}}$ ) which, to the best of our knowledge, has not been reported in the literature for the head and neck. However, these parameters have been described in the evaluation of the kidney [13, 14], liver [15] and pancreas [16], and the $\mathrm{F}_{\mathrm{P}}$ even proved the best parameter to differentiate between normal pancreatic tissue and pancreatic tumour [16]. The current dataset shows that the $\mathrm{F}_{\mathrm{P}}$ was significantly lower in patients with tumours than in those with post-therapeutic changes, possibly because of the presence of leaky inefficient tumour capillaries resulting in a decrease in the fast moving blood pool. The relative diffusion pool is increased because of slowly moving or stagnant blood into capillaries, but diffusion is not increased because of the presence of microvessel membranes [17].

In our prospective study, applied in a well-defined group of 46 patients with laryngeal and hypopharyngeal tumours, conventional MRI with qualitative DW-MRI was highly accurate for the detection of residual/recurrent tumour, even more than the quantitative assessment, which suffered from extensive overlap, limiting its use in the individual patient. However, the subjective nature of visual analysis and the required level of reader expertise (two experienced head and neck radiologists in consensus in this study) make the extrapolation of qualitative data to other centres or applications more difficult. Therefore, both qualitative and quantitative analyses have their place in this setting. Moreover, there have been very promising reports on the usefulness of the ADC values in the prediction of outcome and monitoring of treatment of head and neck squamous cell carcinoma during (chemo)radiotherapy [18-20], which could be even more difficult using visual analysis only. 
Our study has several limitations. Firstly, the functional images were evaluated in conjunction with the morphological images and compared with baseline images, as is usual in clinical practice but induces an element of bias because of the size changes compared with baseline. However, lesions with mass effect are not necessarily always tumour and diffuse swelling can obscure tumour. Secondly, the time interval between the end of (chemo)radiotherapy and imaging was variable as patients in our clinic are not routinely imaged after therapy, but instead are followed clinically every 2 months, with imaging only being performed when patients are symptomatic. This could cause a selection bias, but as we still ended up with more patients without tumour, we believe that this effect is minimal. The absence of surveillance imaging can cause a delay in detection of tumours and therefore its implementation into routine follow-up would be beneficial for the patients. In some patients, the time period between the end of radiotherapy and DW-MRI was relatively long and it can be argued that these patients had second tumours and not recurrent or residual tumours. However, the aim of the study was to differentiate between tumour and post-therapeutic changes, thus, we still included them in the study.

In conclusion, MRI that includes a diffusion-weighted sequence allows detection or exclusion of recurrence in patients treated for laryngeal or hypopharyngeal cancer with a high likelihood, even though four patients had to be excluded from the study due to susceptibility artefacts compromising image quality and its interpretation. This functional imaging technique can easily be added to daily clinical routine provided that good image quality and reader experience are combined with correct correlation with morphological images. Although the results of qualitative image interpretation are excellent and quantitative analysis shows significantly lower ADC values and the perfusion fraction $F_{P}$ being the best parameter for differentiation between recurrent tumour compared with post-therapeutic changes, larger scale studies should be performed in order to improve its application in individual patients.

Acknowledgements Harriet C. Thoeny was supported by a research grant of Carigest SA, representative of an anonymous donor and Majores Foundation, Liechtenstein.

\section{References}

1. Zbären P, Weidner S, Thoeny HC (2008) Laryngeal and hypopharyngeal carcinomas after (chemo)radiotherapy: a diagnostic dilemma. Curr Opin Otolaryngol Head Neck Surg 16:147-153

2. Becker M, Burkhardt K, Allal AS, Dulguerov P, Ratib O, Becker CD (2009) Prä- und posttherapeutische Larynxbildgebung. Radiologe 49:43-58

3. Nömayr A, Lell M, Sweeney R, Bautz W, Lukas P (2001) MRI appearance of radiation-induced changes of normal cervical tissues. Eur Radiol 11:1807-1817
4. Brouwer J, Hooft L, Hoekstra OS et al (2008) Systematic review: accuracy of imaging tests in the diagnosis of recurrent laryngeal carcinoma after radiotherapy. Head Neck 30:889897

5. Brouwer J, Bodar EJ, De Bree R et al (2004) Detecting recurrent laryngeal carcinoma after radiotherapy: room for improvement. Eur Arch Oto Rhino Laryngol 261:417-422

6. de Bree R, van der Putten L, Brouwer J, Castelijns JA, Hoekstra OS, Leemans CR (2009) Detection of locoregional recurrent head and neck cancer after (chemo)radiotherapy using modern imaging. Oral Oncol 45:368-393

7. Vandecaveye V, De Keyzer F, Vander Poorten V et al (2006) Evaluation of the larynx for tumor recurrence by diffusionweighted MRI after radiotherapy: initial experience in four cases. Br J Radiol 79:681-687

8. Zbären P, Christe A, Caversaccio MD, Stauffer E, Thoeny HC (2007) Pretherapeutic staging of recurrent laryngeal carcinoma: clinical findings and imaging studies compared with histopathology. Otolaryngol Head Neck Surg 137:487-491

9. Lell M, Baum U, Greess H et al (2000) Head and neck tumors: imaging recurrent tumor and post-therapeutic changes with CT and MRI. Eur J Radiol 33:239-247

10. Abdel Razek AA, Kandeel AY, Soliman N et al (2007) Role of diffusion-weighted echo-planar MR imaging in differentiation of residual or recurrent head and neck tumors and posttreatment changes. AJNR Am J Neuroradiol 28:1146-1152

11. Thoeny HC (2011) Diffusion-weighted MRI in head and neck radiology: applications in oncology. Canc Imag 10:209-214

12. Vandecaveye V, De Keyzer F, Nuyts S et al (2007) Detection of head and neck squamous cell carcinoma with diffusion weighted MRI after (chemo)radiotherapy: correlation between radiologic and histopathologic findings. Int J Radiat Oncol Biol Phys 67:960-971

13. Thoeny HC, Zumstein D, Simon-Zoula S et al (2006) Functional evaluation of transplanted kidneys with diffusionweighted and BOLD MR imaging: initial experience. Radiology $241: 812-821$

14. Thoeny HC, Binser T, Roth B, Kessler TM, Vermathen P (2009) Noninvasive assessment of acute ureteral obstruction with diffusion-weighted MR imaging: a prospective study. Radiology 252:721-728

15. Yamada I, Aung W, Himeno Y, Nakagawa T, Shibuya H (1999) Diffusion coefficients in abdominal organs and hepatic lesions: evaluation with intravoxel incoherent motion echo-planar MR imaging. Radiology 210:617-623

16. Lemke A, Laun FB, Klauss $M$ et al (2009) Differentiation of pancreas carcinoma from healthy pancreatic tissue using multiple b-values: comparison of apparent diffusion coefficient and intravoxel incoherent motion derived parameters. Invest Radiol 44:769-775

17. Lewin M, Fartoux L, Vignaud A, Arrivé L, Menu Y, Rosmorduc O (2011) The diffusion-weighted imaging perfusion fraction $\mathrm{f}$ is a potential marker of sorafenib treatment in advanced hepatocellular carcinoma: a pilot study. Eur Radiol 21:281290

18. King AD, Mo FK, Yu KH et al (2010) Squamous cell carcinoma of the head and neck: diffusion-weighted MR imaging for prediction and monitoring of treatment response. Eur Radiol 20:2213-2220

19. Vandecaveye V, Dirix P, De Keyzer F et al (2012) Diffusionweighted magnetic resonance imaging early after chemoradiotherapy to monitor treatment response in head-and-neck squamous cell carcinoma. Int J Radiat Oncol Biol Phys 82:1098-1107

20. Thoeny HC, Ross BD (2010) Predicting and monitoring cancer treatment response with diffusion-weighted MRI. J Magn Reson Imaging 32:2-16 